\title{
DIAGNOSING OF ROTOR SYSTEMS OF MARINE GAS TURBINE ENGINES IN NONSTATIONARY STATES
}

\author{
Andrzej Grządziela \\ Polish Naval Academy \\ Śmidowicza Street 69, 81-103 Gdynia, Poland \\ tel.: +48586262724 \\ e-mail:a.grzadziela@amw.gdynia.pl
}

\begin{abstract}
Vibration tests of marine gas turbine engines are performed as researches on-line and off-line types. Systems online type generally monitored one or two vibration symptoms, which asses the limited and/or the critical values of parameters and they potentially can warn and/or shutdown engines. Systems off-line types are usually used for vibration analyzing during non-steady state of work. The paper presents comparison of different methods of analyzing vibration symptoms measured under run-up and shut-down processes of marine gas turbine engines. Results of tests were received on gas turbine engine DR76 type of the COGAG type propulsion system. The engine was not fitted with vibration monitoring system. Main goal of researches was qualified of helpfulness and unequivocally results received by methods of synchronous measurement, order tracking and auto tracking. All vibration symptoms were chosen from the methodology of the diagnosing gas turbine engines operated in the Polish Navy, called BDS (Base Diagnosing System). Second purpose was estimation of the possibility of implementation presented methods of vibration analyses of gas turbine engines for on-line monitoring system. Application of periodical or on-line diagnostic procedures makes it possible to operate ship propulsion systems in accordance with their current technical state. The realization of the investigations made reliable verification of the investigation results possible.
\end{abstract}

Keywords: dynamics, gas turbines, rotor vibration, run-up process

\section{Introduction}

Operation of naval ships propulsion systems is a complex task due to specific features of marine environment as well as demand of maintaining high level of serviceability and reliability of the ships. Application of periodical or on-line diagnostic procedures makes it possible to operate ship propulsion systems in accordance with their current technical state. Especially, in the case when gas turbines engine maintenance schedule is a criterion for maintenance time determination. Though such exploitation strategy makes early scheduling of maintenance operations and their logistic assurance possible, but it simultaneously contributes to increase of costs because of its replacement system of elements (technically often still serviceable ones) as well as it makes impossible to early detect primary symptoms of failures occurring before the end of maintenance time.

Application of the vibration diagnosing makes managing the engine's operation times much more rational, especially at its end and does not require taking the ships out of service. It is possible to divide vibrations measurements of marine gas turbine engines on:

- off-line type (measurements made as a free run or synchronous sampling),

- on-line type (real time monitoring).

Both methods have their own advantages and disadvantages. Off-line type systems are usually offered as very simple analyzers - data collectors. Defined measuring path allows in a vessels power plant, with programmed setups of registration, using of average technical personnel, which the main task is precision in measuring procedure. Analysis of measured data proceeds beyond the shipping power plant, on the properly equipped laboratory stand. There are not wide population of data collectors off-line types enable to analyze vibration in real time and sign the outrun of limited values of diagnosing symptoms. The main advantage of this kind of devices is the prize, but it is 
necessary to underline that data collectors are mainly useful only for assessing the vibro-activity of gas turbine engines.

The vibration, diagnostics systems on-line type ensures permanent supervision of the technical state of gas turbine engines, including recording, analyzing, alarming and predicting. It allows recognizing primary symptoms of the permanent, technical state changing with possibility of the trend remarks. Vibration systems on-line type usually works as an element of the complex, multisymptoms, diagnosing system of vessels power plant.

Diagnosing such machines like gas turbine engines depends on measurement and processing of vibration signals. It is an important fact that marine gas turbine engines do not work with a constant rotational speed of compressors and power turbines rotors. That is the reason to synchronize processing method with selected reference magnitude that is rotational frequency of one of rotors $[2,3]$. It allows recognizing most of the typical multifunction of rotors systems. Basic, operational inefficiency of gas turbine engines include following question:

- in result of damage or crushing of the compressors' blade(s) or power turbines blade(s) - more rarely,

- in result emerged unbalancing coming from the carbon deposit or the salinity,

- in result of seizing of rotors sealing system and leaking the lubrication oil inside the rotor,

- in result of aero-dynamical misalignment between gas generator and power turbine,

- in result of thermal damages of combustion chambers - torsional vibration of the power turbine,

- in result of damage auxiliary mechanisms of the engine.

Some of inefficiency can be recognized in the vibration spectra as a change of natural frequency of rotated elements of engine, so it is a reason to introduce the synchronous sampling under variable engine condition, e.g. run-up or shut-down processes. The occurrence of non-stationary effects, typical in the case of small unbalancing, can also be the result of progressive forming of damages, even though their intensity is slight in the early stages. Results presented in the paper compare different synchronous methods of vibrations analyzing and discuss on obtained results similar like the PLD method [7]. The presented method can be introduced to the gas turbine monitoring systems as an early predictive diagnostics tool of unbalance identification.

\section{Object of investigation}

Monitoring vibration signals coming from rotated machines is a common diagnostics procedure, well known all over the world [2, 5, 7]. Most of rotated machines like marine gas turbine engines are constructed as supercritical machines so analyzing of vibration parameters in the steady state are technically limited. Therefore it was decided to analyze rotors dynamics of marine gas turbine engines in non-steady states using off-line method. It was expected to take information on the following areas: rotor unbalancing, rotors misalignment, natural frequencies, critical speeds etc.

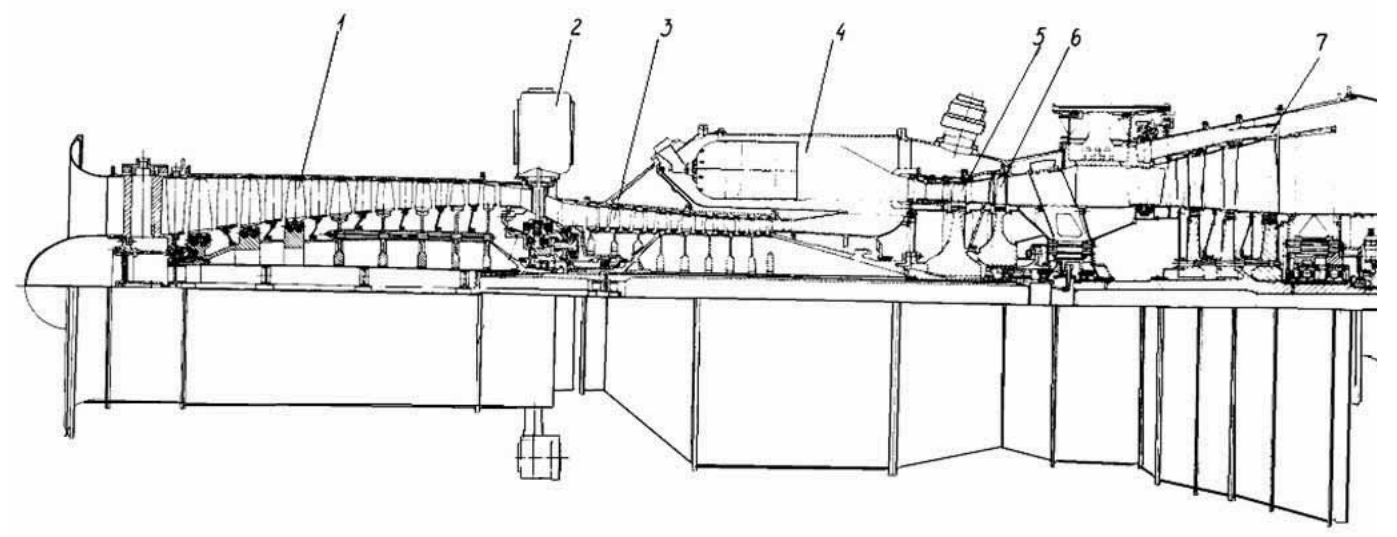

Fig. 1 Longitudinal section of rotor system gas turbine engine DR76 type, where: 1 - low pressure compressor (LPC), 2 - auxiliary drives, 3 - high pressure compressor (HPC), 4 - burning chambers, 5 - high pressure turbine (HPT), 6 - low pressure turbine (LPT), 7 - power turbine (PT) 
The object of researches was a marine gas turbine engine DR76 type mounted in the COGAG type propulsion plant of Polish Navy Tarantula class corvette. The scheme of longitudinal section of rotors system presents Fig. 1. The investigation presented below covered analyzes of vibration velocity (order tracking) during run-up and shut-down processes in the turning of engine process.

\section{Non - steady states vibration signals analysing}

For realization of the first type of measurements the analyzer of Brïel \& Kjær 3560B was used, while collecting and processing of measured data were made possible by using the program PULSE [8]. Two measuring transducers (accelerometers ICP type) were fixed to steel cantilevers located on the flange over the front strut of the LPC. The fixing cantilevers characterized of a natural vibration resonance frequency value different enough from harmonic frequencies due to rotation speed of the given rotors so it is an important of the recording procedure. The measurements were taken perpendicularly to the rotation axis of the rotors. Such choice was made on the basis of theoretical consideration of excitations due to unbalanced shaft rotation, and results of preliminary investigations of the object $[2,4,7]$.

\subsection{Vibration analyzing of the run-up process}

First test was analyzing the run-up process in the turning of engine process, often called the cold start process. The cold start process is useful for vibration analyses due to the elimination disturbances from the combustion process. Characteristic of acceleration of the LPC rotor presents Fig. 2.

Synchronous signal was collected from the tachometer coupled with the auxiliary drive gear box where the transmission ratio average $i=0.125$, so the LPC rotor had 8 times higher rotational speed than presented in the Fig. 2. The main goal of the synchronous analyzing vibration signals during the run-up process was the identification of dynamics disturbances. The influence of "other" signals is presented in the Fig. 3.

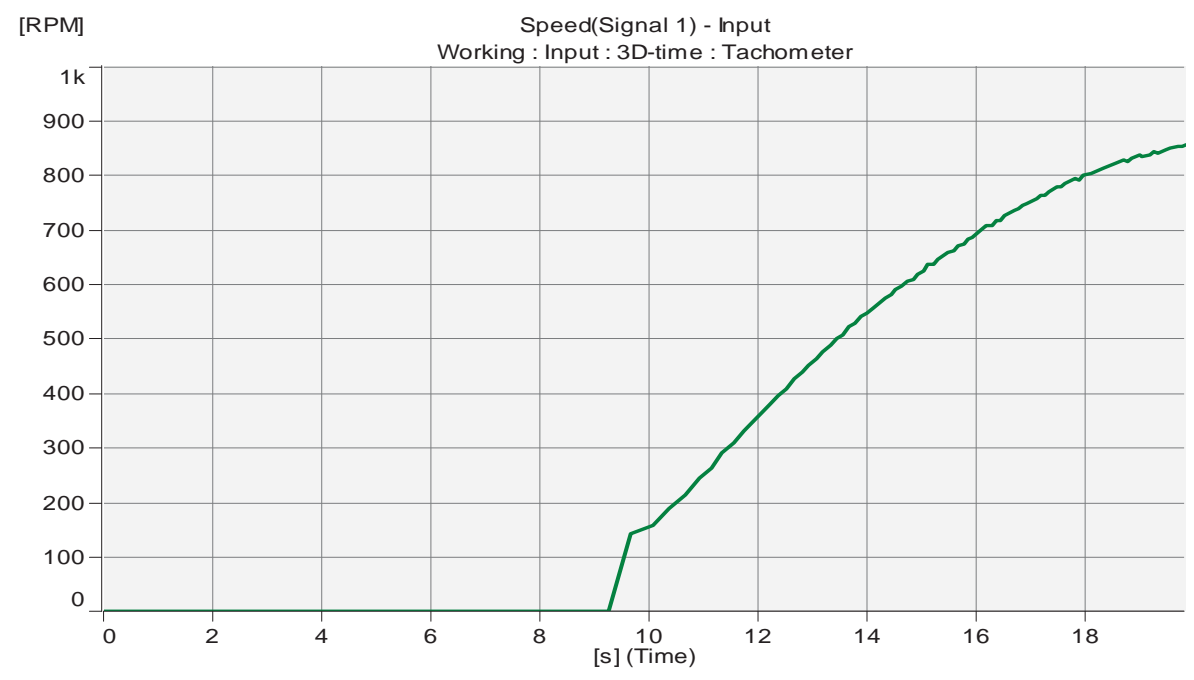

Fig. 2. Rotors LPC rotational speed characteristics during run-up process

The run-up process was started in point $t=7$ second (see Fig. 3) so all vibration signals recorded before start point contain "other" signals coming from dynamics of another rotated machines in the power plant, natural frequencies of engine or their combinations. It allows identifying main "other" signals like: $\mathrm{f}_{1}=305 \mathrm{~Hz}, \mathrm{f}_{2}=600 \mathrm{~Hz}, \mathrm{f}_{3}=1,6 \mathrm{kHz}$ i f $\mathrm{f}_{4}=2 \mathrm{kHz}$ which originate from the natural frequencies of the engine foundation. The most energetic signal in the domain of the velocity of vibration, recorded during rotors acceleration, is the I harmonic. 


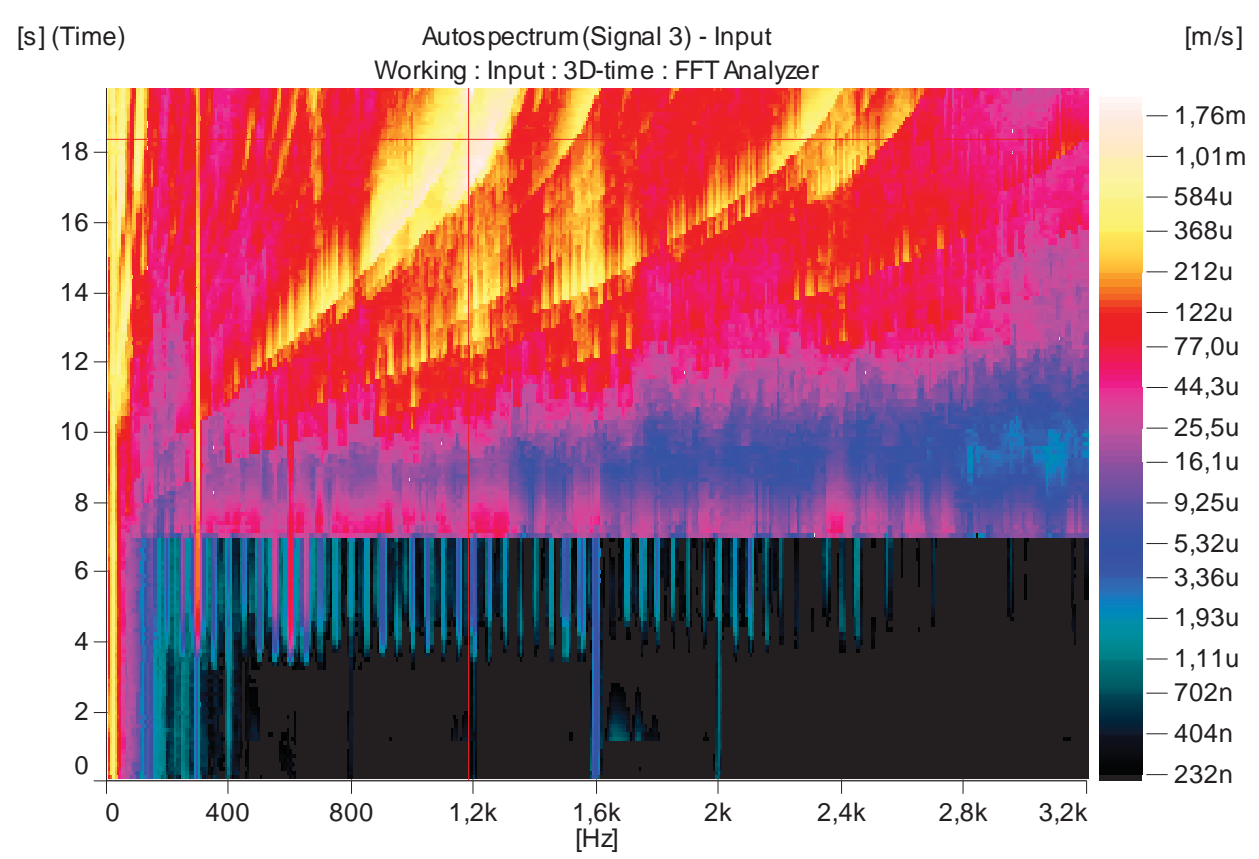

Fig. 3. Synchronous spectra of the velocity of vibration during run-up process with using the band - pass filter of $0.1 \mathrm{~Hz}-3.2 \mathrm{kHz}$ range

The synchronous spectra recorded after $t=7$ seconds confirms the difficulty of the unambiguous identification of the spectral components including I harmonic as well.

\subsection{Vibration analyzing of the shut-down process}

Next test, connected with analyzes of vibration parameters, was the shut-down processes in the turning of engine process. The Order Tracking lies in the fact that tacho signal delivering the tracking reference must be selected from rotating shaft. Tacho, speed level and speed interval triggers are available when a

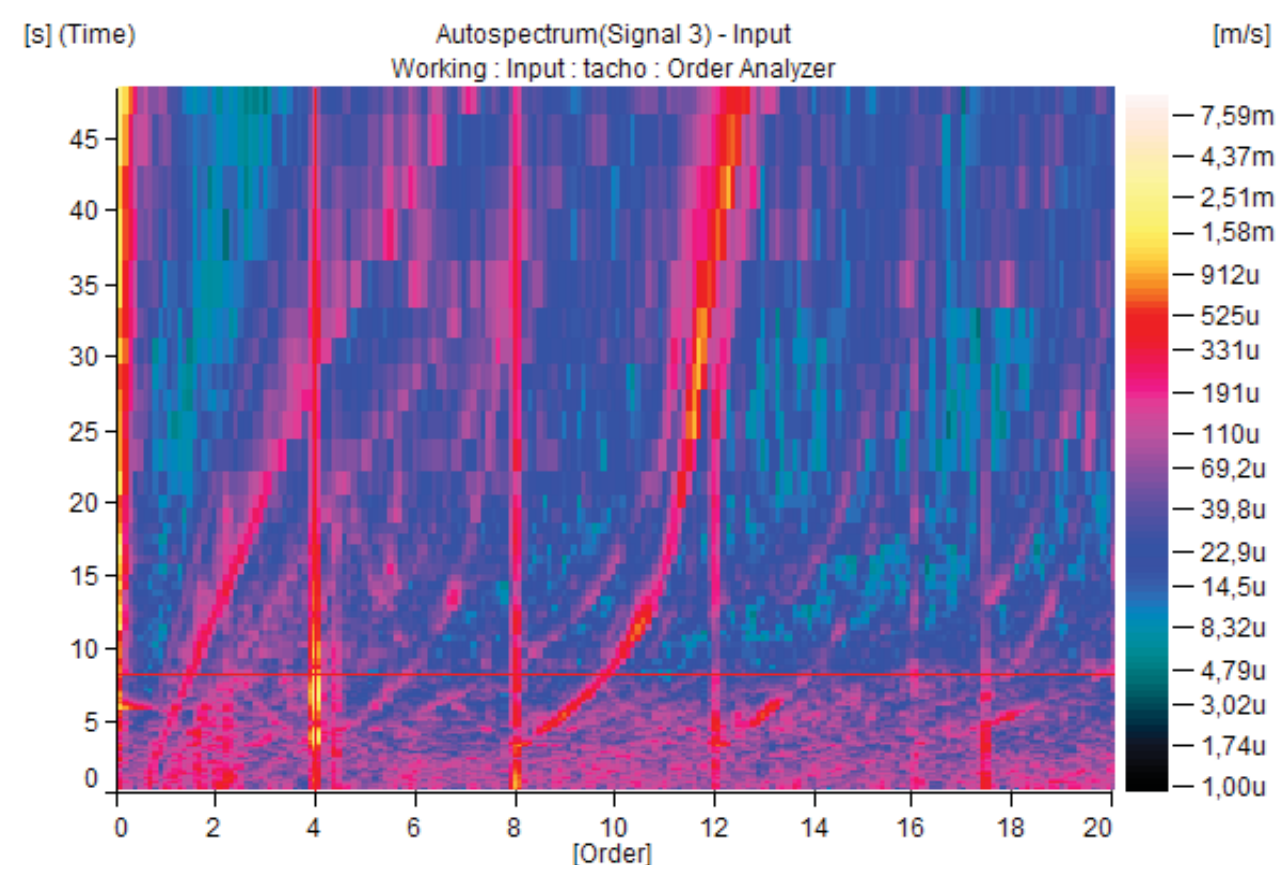

Fig. 4. Autospectrum of velocity of vibration in the shut-down process with the use of order tracking procedure, in the domain of time function 
Tachometer is inserted in a measurement setup. They can be used for triggering measurements and starting, updating and/or stopping storage of time signals and/or spectra to multi-buffers. Order analyzers can measure time (revs) signals, autospectra for signals and the cross-spectra of selected pairs of signals.

The Fig. 4 presents autospectrum of signal 3 of velocity of vibration with the use of Order Tracking procedure [8]. Changes of parameters are presented in the domain of time function - Fig. 4. In contrary to run-up process the main vibration signal in the shut-down process of rotor is subharmonic (1/2 of harmonic) - presented as a 4 Order. Gradient slope of pressure characteristic of the lubrication oil in the radial bearing brought the upgrowth the misalignment between of HPC and LPC rotors (HPC shaft rotated inside the LPC shaft - see Fig. 1) and typical in the technical diagnostics domination of subharmonic.

Upgrowth of stiffness of bearing system confirm the "right-hand branches" of harmonics appeared from the time point equal 4 second from 4,8 and 12 orders when the pressure of lubrication oil fall down.

Analyses of gas turbine rotors dynamics in the non-steady states with the use of PULSE system should be made in both processes. The start-up process allows recognizing "other" signals but the identification of dynamics features is very difficult regard to the high rotors acceleration. Identification of dynamics features of rotors system is well recognized in deceleration as main orders characteristics - Fig. 5 and 6. Both characteristics confirm results of modes of the rotor system calculated with the use of FEM [6].

Analyze of the I harmonic (8 order) enables to observe changes of dynamics features as a trends. Application of the domain of rotors rotational speed function is the most important thing in basic orders analyze during shut-down process. It allows identifying changes of natural frequencies without any disturbances signals coming from thermo- dynamics processes typical for "hot work" of gas turbine engine - Fig. 5.

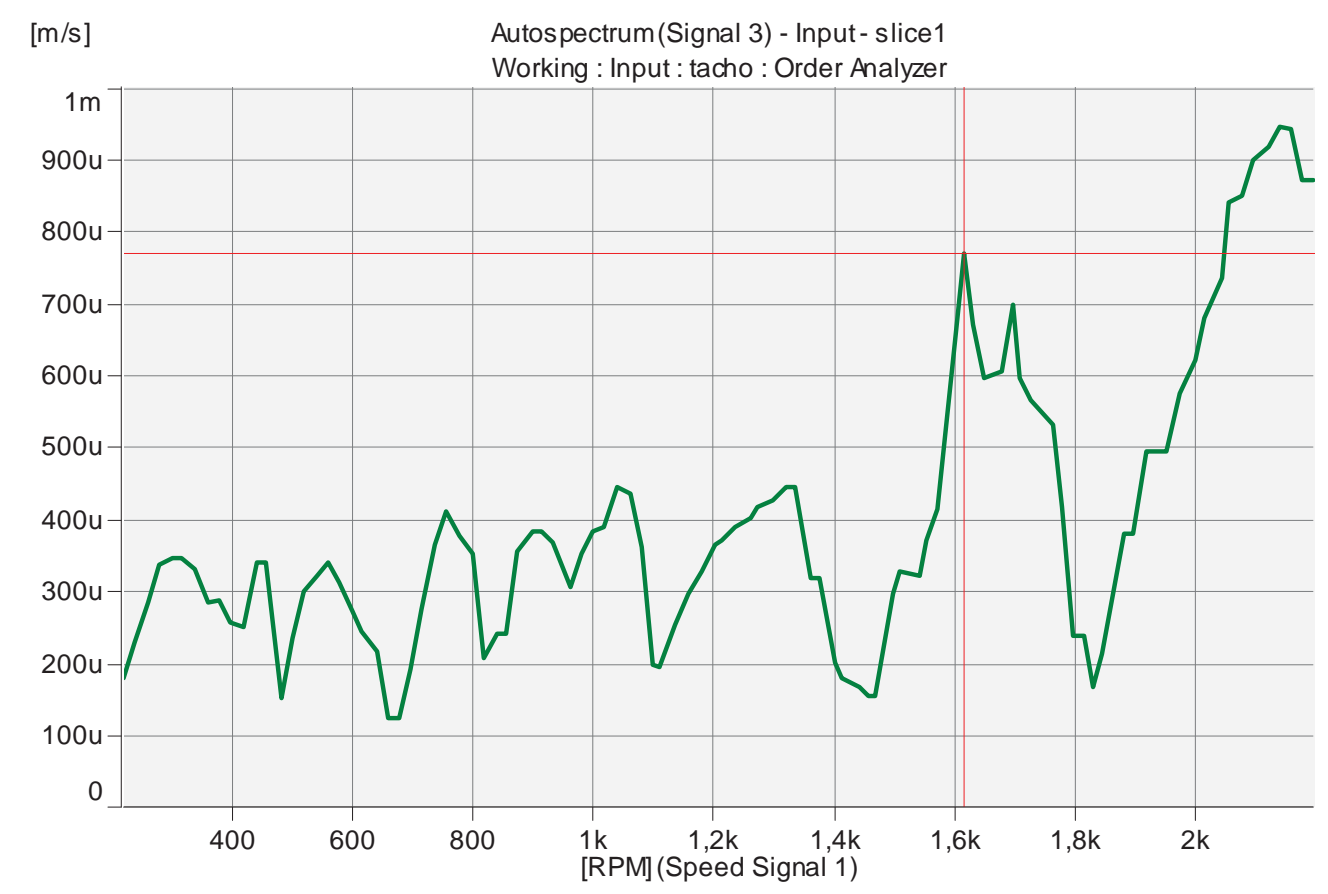

Fig. 5. Autospectrum of 8 order (I harmonic) of velocity of vibration in the shut-down process of LPC rotor stoppage

Analyzes of subharmonics signals are very useful as well. The autospectrum of 4 order (subharmonic) of velocity of vibration in the shut-down process of LPC rotor shows individual features of analyzed rotor system. Increase of subharmonic value in the domain of rotors rotational speed is like a fingerprints of rotor system. 
Every changes of technical state like changes of stiffness and damping, unbalancing or misalignment caused changes in the characteristic of subharmonic - Fig. 6.

\section{Conclusions}

The realization of the investigations with the use of Order Tracking procedure made reliable verification of the investigation results possible. The following detail conclusions were drawn for further diagnostic inference of compared method:

- both programs - start up and stoppage, have their own advantages and disadvantages but they can fulfil all technical requirements for diagnosing rotors systems of marine gas turbine engines,

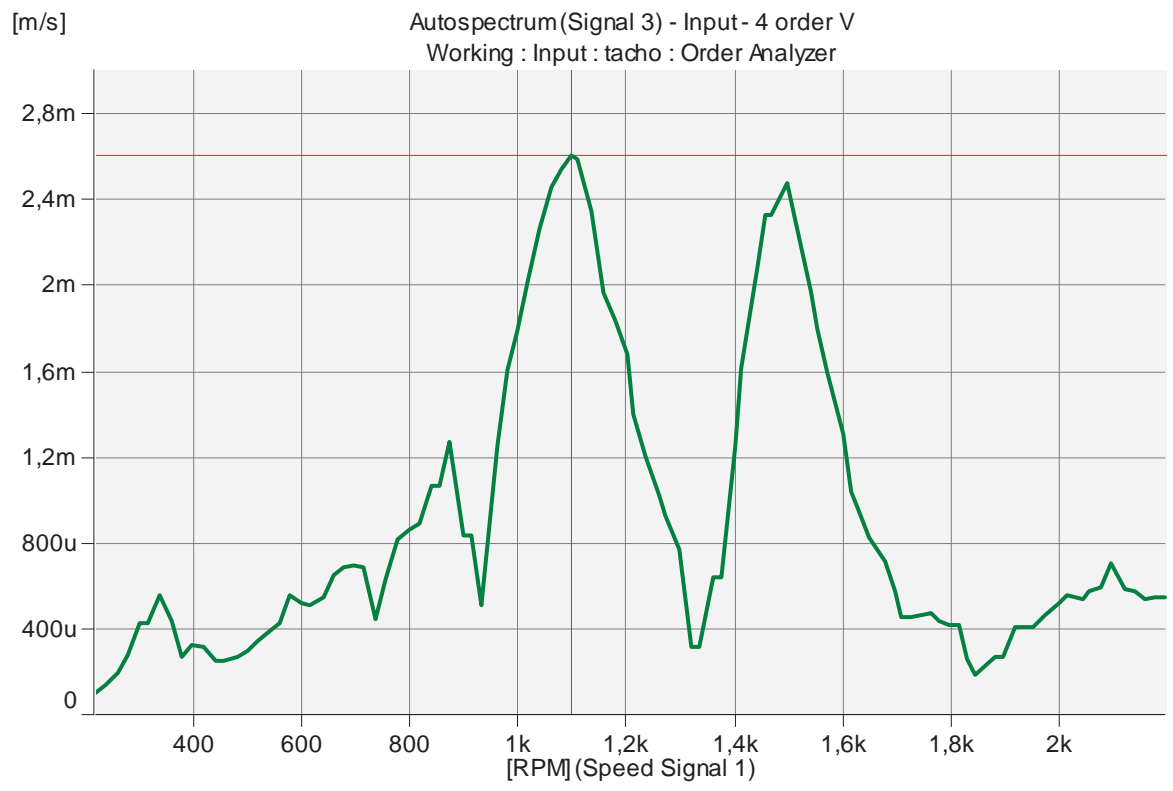

Fig. 6. Autospectrum of 4 order (subharmonic) of velocity of vibration in the shut-down process of LPC rotor stoppage

- the synchronized measurement of vibration signals allows recognizing specified symptoms of resonance and changes of natural frequencies during processes of run-up and stoppage of rotor systems,

- application Order Tracking procedures for monitoring system of gas turbine engines enables recognize changes of technical state characteristic for modes changes. Identification of foundation stiffness changing or unbalancing coming from impurities of the rotor system implicates natural frequencies changing which are observed in Order Tracking waterfall charts.

\section{References}

[1] Cioch, W., Jamro, E., Digital signal acquisition and processing in FPGAs. Przegląd Elektrotechniczny, Stowarzyszenie Elektryków Polskich, Main topics: Electrical measurements, R 85, Nr 2, 2009.

[2] Charchalis, A., Grzadziela, A., Diagnosing of naval gas turbine rotors with the use of vibroacoustic parameters, The 2001 International Congress and Exhibition on Noise Control Engineering, The Hague, The Netherands, p. 268, 2001.

[3] Downham, E., Woods, R., The rationale of monitoring vibration on rotating machinery, ASME Vibration Conference, Paper 71 - Vib - 96, 1971.

[4] Grządziela, A., Vibroacoustic method of shafting coaxiality assessment of COGAG propulsion system of a vessel, Polish Maritime Researches, No. 3, pp. 29-30, 1999. 
[5] Grządziela, A., Diagnosing of naval gas turbine rotors with the use of vibroacoustics parameters, Polish Maritime Researches, No. 3, pp. 14-17, Gdańsk 2000.

[6] Grządziela, A., Vibration analysis of unbalancing of marine gas turbines rotors, Mechanika, T. 23, Z. 2, pp. 187-194, 2004.

[7] Krzyworzeka, P., Adamczyk, J., Cioch, W., Jamro, E., Monitoring of nonstationary states in rotating machinery, ITE, Kraków - Radom 2006.

[8] Pedersen, T. F., Gade, S., Harlufsen, H., Konstantin-Hansen, H., Order tracking in Vibroacoustic Measurements: A Novel Approach Eliminating the Tacho Probe, Technical Review, Brüel \& Kjær, No. 1, pp. 15-28, 2006. 